\title{
Global Isotope Metabolomics Reveals Adaptive Strategies for Nitrogen Assimilation
}

\author{
Michael E. Kurczy ${ }^{\dagger}$, Erica M. Forsberg ${ }^{\dagger}$, Michael P. Thorgersen $n^{\ddagger}$, Farris L. Poole $I^{\ddagger}$, H. Paul \\ Benton $^{\dagger}$, Julijana Ivanisevic ${ }^{\S}$, Minerva L. Tran ${ }^{\dagger}$, Judy D. Wall ${ }^{\perp}$, Dwayne A. Elias ${ }^{\|}$, Michael W. \\ W. Adams ${ }^{*}, \neq$, and Gary Siuzdak ${ }^{*},+\#$
}

†Scripps Center for Metabolomics, The Scripps Research Institute, 10550 North Torrey Pines Road, La Jolla, California 92037, United States ¥Department of Biochemistry and Molecular Biology, University of Georgia, Athens, Georgia 30602, United States §Metabolomics Platform, Faculty of Biology and Medicine, University of Lausanne, Rue du Bugnon 19, 1011 Lausanne, Switzerland "Biosciences Division, Oak Ridge National Laboratory, Oak Ridge, Tennessee 37831, United States ${ }^{\perp}$ Department of Biochemistry, University of Missouri, Columbia, Missouri 65211, United States "Departments of Chemistry, Molecular, and Computational Biology, The Scripps Research Institute, 10550 North Torrey Pines Road, La Jolla, California 92037, United States

\section{Abstract}

\begin{abstract}
Nitrogen cycling is a microbial metabolic process essential for global ecological/agricultural balance. To investigate the link between the well-established ammonium and the alternative nitrate assimilation metabolic pathways, global isotope metabolomics was employed to examine three nitrate reducing bacteria using ${ }^{15} \mathrm{NO}_{3}$ as a nitrogen source. In contrast to a control (Pseudomonas stutzeri $\mathrm{RCH} 2)$, the results show that two of the isolates from Oak Ridge, Tennessee (Pseudomonas N2A2 and N2E2) utilize nitrate and ammonia for assimilation concurrently with differential labeling observed across multiple classes of metabolites including amino acids and nucleotides. The data reveal that the N2A2 and N2E2 strains conserve nitrogen-containing metabolites, indicating that the nitrate assimilation pathway is a conservation mechanism for the assimilation of nitrogen. Co-utilization of nitrate and ammonia is likely an adaption to manage higher levels of nitrite since the denitrification pathways utilized by the N2A2 and N2E2 strains from the Oak Ridge site are predisposed to the accumulation of the toxic nitrite. The use of global isotope metabolomics allowed for this adaptive strategy to be investigated, which would otherwise not have been possible to decipher.
\end{abstract}

\footnotetext{
*Corresponding Authors: Tel.: (706) 542-2060. adamsm@uga.edu. Internet: http://adams.bmb.uga.edu/. Tel.: (858) 784-9425. siuzdak@scripps.edu. Internet: https://masspec.scripps.edu/.

Author Contributions

The authors would like to acknowledge joint co-first authorship of this article to Michael E. Kurczy, Erica M. Forsberg, and Michael P. Thorgersen.

Notes

The authors declare no competing financial interest.

Supporting Information

The Supporting Information is available free of charge on the ACS Publications website at DOI: 10.1021/acschem-bio.6b00082.

Metabolite identification confirmation via MS/MS database matching and control experimental data (PDF)
} 


\section{Graphical Abstract}

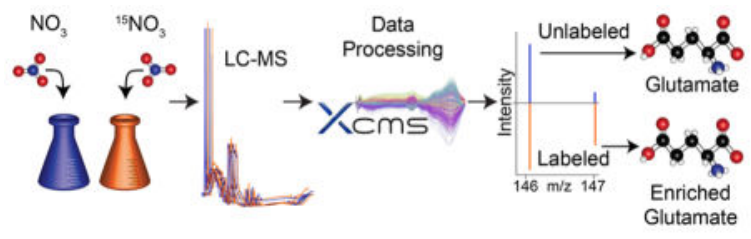

The microbial communities responsible for the modern nitrogen cycle came into existence approximately 2.5 billion years ago and evolved over this time to fine-tune the balance between molecular and fixed nitrogen. Industrial nitrogen fixation in contrast has dramatically outpaced evolution to upset this balance in just over a century's time. ${ }^{1-3}$ The inability of microbes to sufficiently clear (denitrify) this biologically available nitrogen has been linked to eutrophication and hypoxia in coastal and terrestrial waters. ${ }^{4-7}$ Nitrogen is also fundamental to cellular metabolism and essential for microbial growth. The balance between anaerobic respiration (denitrification) and assimilation of nitrogen is key to understanding the fitness of the microbes that carry out this critical part of the nitrogen cycle. ${ }^{8}$ Nitrate, a highly oxidized form of nitrogen, is used by denitrifying microorganisms as an electron acceptor and is reduced to nitrogen gas over several steps to close the nitrogen cycle. A more reduced form of nitrogen, ammonium, is utilized for assimilation to create a multitude of biomolecules. However, there is an increasing body of work showing that nitrate assimilation is a conserved pathway across many bacteria and archaea and is relevant in various environments. ${ }^{9,10}$ Generally in soil microorganisms, ammonium is used preferentially rather than nitrate as a nitrogen source. ${ }^{11,12}$ However, in Enter-obactericeae and Pseudomonas species, under nitrogen limited conditions, the nitrate assimilation genes are activated through a signal cascade involving the two-component regulatory system NtrBC. ${ }^{11,13}$ In this particular study, we are interested in comparing the metabolomes of N2A2 and N2E2, a unique pair of Pseudomonas strains isolated from a pristine site in Oak Ridge, Tennessee, and Pseudomonas stutzeri $\mathrm{RCH} 2$ isolated from a contaminated site in Hanford, Washington. Both N2A2 and N2E2 appear to lack regulatory control of nitrate assimilation even though they contain homologues of the NTR system for nitrate assimilation regulation, ${ }^{14}$ while $\mathrm{RCH} 2$ responds with the expected behavior of preferentially assimilating ammonium over nitrate. This experiment was conceived to follow up on the finding that the metabolomes of the Oak Ridge strains were more or less unchanged following the removal of ammonia.

To examine this phenomenon, we employed global metabolomics, which provides a survey of the total metabolome and helps identify activated biochemical pathways in specific phenotypes. Methodologies used for global metabolomics include Nuclear Magnetic Resonance (NMR) ${ }^{15-17}$ and gas chromatography (GC) ${ }^{18-20}$ or liquid chromatography mass spectrometry (LC/MS) ${ }^{21-23}$ A typical workflow for untargeted LC/MS-based metabolomics involves several steps. First, metabolites must be extracted from the sample and chromatographically separated. A more popular chromatographic technique that has recently advanced is hydrophobic interaction liquid chromatography (HILIC). This method has the power to separate polar and even nonpolar analytes. ${ }^{24}$ HILIC typically has excellent 
coverage for biologically relevant metabolites in comparison to typical reverse phase chromatography that tends to favor nonpolar metabolites. Mass spectral data are then collected for samples in each sample group using high resolution mass spectrometry, often employing QTOF or Orbitrap technology ${ }^{25}$ in order to improve accuracy in putative metabolite identification. Next, the data are processed by one of the available metabolomics analysis platforms such as Metabolic profiler (Bruker), Simca-P (Umetrics), Markerlynx (Waters), Mass Profiler Pro (Agilent), MetAlign, ${ }^{26}$ MZmine, ${ }^{27}$ MAVEN, ${ }^{28}$ MetaboAnalyst, ${ }^{29}$ and $\mathrm{XCMS}^{30}$ and XCMS Online ${ }^{31,32}$ to identify the features that significantly change between sample groups. The significantly altered features are then compared to metabolite databases such as METLIN, ${ }^{33,34} \mathrm{KEGG},{ }^{35} \mathrm{HMDB},{ }^{36}$ or $\mathrm{MMCD}^{37}$ and given a putative identification. The identities of the metabolites are then validated by comparison with standards and by tandem MS (MS/MS).

To facilitate these analyses, stable isotopes were used in conjunction with global metabolomics since isotopic labeling has historically been a very powerful tool for understanding metabolic pathways. ${ }^{38-40}$ Isotope pathway analysis is typically performed in a targeted manner where a pathway is proposed to be associated with a given phenotype. An isotopically labeled substrate that is known or suspected to be involved in that pathway is introduced into the system and then monitored for the presence and/or quantity of specific downstream metabolites. These studies often require significant knowledge of the metabolic pathways and metabolite compounds involved and require intensive isotope distribution ratio analyses, particularly in the case of kinetic flux experiments. ${ }^{41,42}$ This research strategy is well suited to confirm hypotheses and not commonly used as a preliminary investigational research tool. High resolution mass spectrometry, however, allows for the analysis of thousands of metabolite features that are unseen by other technologies. When used in combination with isotope labeling, it facilitates the tracking of many substrates by the presence of metabolite isotopomers without previous knowledge of the pathways involved. To handle the unique data processing needs of these information dense data sets, the Patti lab has released $\mathrm{X}^{13} \mathrm{CMS},{ }^{43}$ an open source bioinformatics tool capable of identifying many isotopically enriched metabolite features to track metabolism in an unbiased manner. This software can be used in conjunction with isoMETLIN ${ }^{44}$ (http://isometlin.scripps.edu), a version of the METLIN database that currently contains mass to charge ratios and MS/MS data for isotopically labeled metabolites. Here, we utilize ${ }^{15} \mathrm{~N}$ stable isotope global metabolomics to investigate the link between denitrification and nitrogen assimilation, first by analysis of the global metabolomics, followed by an in-depth analysis of the assimilatory nitrate reduction to ammonia (ANRA) pathway with the incorporation of ${ }^{15} \mathrm{~N}$ stable isotope global metabolomics.

\section{RESULTS AND DISCUSSION}

\section{Global Metabolomics of RCH2}

Nitrate assimilation is typically suppressed by the presence of ammonia; energetically this is logical as ammonia is the end product of this multistep enzymatic reduction in a number of microbes. However, there might be environmental situations where the assimilatory nitrate reduction to ammonia is advantageous regardless of the additional energy cost. To 
understand the metabolic consequences of utilizing the nitrate assimilation, we grew the well-characterized model denitrifier Pseudomonas stutzeri $\mathrm{RCH} 2$ with $10 \mathrm{mM} \mathrm{KNO}_{3}$ in the presence and absence of $5 \mathrm{mM} \mathrm{NH}_{4} \mathrm{Cl}$.

The global metabolomic LC/MS analysis was carried out by HILIC to facilitate the identification of central carbon metabolites. Negative mode was chosen as it has the ability to ionize a broad range of polar metabolites, although some classes of metabolites, such as amino acids, may be attenuated compared to positive mode. ${ }^{45}$ The cloud plot (Figure 1a) shows hundreds of affected features. Each feature is an ion detected at a given retention time and plotted as a circle on the cloud plot where the diameter scales with the fold change and color depth represents a lower $p$ value. It is important to mention that while the number of dysregulated features indicates that the metabolism of $\mathrm{RCH} 2$ is altered under ammonia free conditions, these are not metabolites per se. To identify the metabolites, it is necessary to provide orthogonal information such as tandem MS (MS/MS) for each precursor ion (Supporting Information, Table 1).

Using targeted MS/MS and the MS/MS match feature (Supporting Information Table 1) in the METLIN database, we were able to positively identify 29 metabolites. The identified metabolites are presented in a heat map (Figure 1b). The first observation was that the two classes $\mathrm{RCH} 2$ and $\mathrm{RCH} 2-\mathrm{NH}_{4}$ tended to cluster based on the signal intensities of the identified metabolites. The metabolites affected were, in general, nitrogen-containing molecules. For example, we see that amino acids are largely decreased under ammonia free conditions. This is expected as nitrogen can enter the metabolome via the synthesis of glutamate. This takes place by the action of glutamate dehydrogenase, which assimilates ammonia to $a$-ketoglutarate. Glutamate then in turn undergoes transamination to provide ammonia for other amino acids. The lack of glutamate is correlated to an increase in the $a$ ketoglutarate showing that the production of ammonia via nitrate assimilation is not sufficient to maintain glutamate production.

Likewise, we also found that the purine and pyrimidine synthesis pathways were disrupted. In general, we observed that these nitrogen-containing compounds were down regulated. This again can be traced back to the lack of amino acid synthesis, as this is ultimately the nitrogen source for de novo purine/pyrimidine synthesis. Surprisingly, we find that the diand triphosphorylated nucleotides actually increase in the ammonia-free conditions; this may be a consequence of the new energy requirement associated with the use of nitrate as a nitrogen source.

\section{Global Metabolomics of N2A2 and N2E2}

The cloud plots representing analysis of N2A2 and N2E2 (Figure 2) show that there is very little difference in the metabolite profile when ammonia is not present. This is in stark contrast (Figure 1) to the RCH2 strain where several metabolic pathways appear to be disrupted. It is apparent by these results that the strains isolated from the Oak Ridge Reservation as well the $\mathrm{RCH} 2$ strain have the ability to assimilate $\mathrm{NO}_{3}$. However, it was not clear how these cells utilized nitrogen sources when both ammonia and nitrate were available. Certainly, the Oak Ridge cells were utilizing nitrate for ANRA even in the presence of ammonia, but it was not apparent if they were also utilizing ammonia under 
these conditions. Furthermore, $\mathrm{RCH} 2$ is capable of assimilating $\mathrm{NO}_{3}$ via ANRA, but it was not clear if this pathway was being accessed in the presence of ammonia. The answers to these questions were obscured within the metabolome but could be revealed by global ${ }^{15} \mathrm{~N}$ stable isotope metabolomics.

\section{Global $\left({ }^{15} \mathrm{~N}\right)$ Isotope Metabolomics}

The growth conditions used to understand the utilization of nitrogen sources is described (Figure 3a). The cells were grown up in $5 \mathrm{mM}$ ammonia and $10 \mathrm{mM}$ nitrate. In one batch the nitrate was labeled with ${ }^{15} \mathrm{~N}$ so that any assimilated nitrate would manifest as isotopically labeled metabolites. It should be noted that the centrifugation step may have an effect on metabolism prior to quenching, which can alter high flux metabolites on shorter time scales. ${ }^{42}$ However, under these conditions, the cells should have a sufficient nitrogen source to reach steady state and sufficient ammonia to use for assimilatory purposes, and thus it was expected that the nitrate would be used as an electron acceptor and reduced to $\mathrm{N}_{2}$ in the case of the $\mathrm{RCH} 2$ and $\mathrm{N} 2 \mathrm{~A} 2$ strains and to nitrite in the case of the N2E2 strain, which lacks dissimilatory nitrite reductase.

As expected, we found that the scenario described above is true for $\mathrm{RCH} 2$. Using global metabolomics, we find virtually no difference in the metabolome of the ${ }^{15} \mathrm{~N}$ labeled vs unlabeled (Supporting Information Figure 1). That is to say there are no ${ }^{15} \mathrm{~N}$ labeled metabolites and therefore no nitrate assimilation through ANRA in the presence of ammonia. A lack of ${ }^{15} \mathrm{~N}$ labeling is depicted (Figure $3 \mathrm{~b}$ ) by the isotope pattern for glutamate extracted from the labeled $\mathrm{RCH} 2$ that is identical to the mirrored isotope pattern from the unlabeled batch.

The presence of ammonia in the $\mathrm{RCH} 2$ growth media has blocked the ANRA pathway (Figure $3 \mathrm{c}$ ). The ${ }^{15} \mathrm{~N}$ labeled nitrate is reduced to $\mathrm{N}_{2}$ as readily available ammonia is used for assimilation. This indicates that ammonia suppresses ANRA by blocking assimilatory nitrite reductase. The ANRA pathway begins with the transport of nitrate into the cytosol where it is reduced to nitrite; the nitrite is then reduced to ammonia in one step by assimilatory nitrite reductase. If there is sufficient product (ammonia), the enzyme function is inhibited, allowing any nitrite that has been formed to take part in denitrification. In the denitrification pathway, nitrite will first be reduced to nitric oxide, followed by nitrous oxide and then finally to nitrogen gas. Thus, using ${ }^{15} \mathrm{~N}$ labeled nitrate and global isotope metabolomics, we have shown that $\mathrm{RCH} 2$ uses ammonia when present for assimilation exclusively while nitrate is utilized for denitrification and only accesses the ANRA pathway under ammonia-free conditions (Supporting Information Figure 2).

On the other hand, we find that the N2A2 and N2E2 strains isolated from Oak Ridge do assimilate nitrate in the presence of ammonia. This is represented by the isotope ratios of glutamate in ${ }^{15} \mathrm{~N}$ labeled vs unlabeled samples (Figure $3 \mathrm{~b}$ ). The top mass spectrum shows the isotope distribution from the unlabeled cells, which matches the predicted isotope ratio for glutamate where the ${ }^{13} \mathrm{C}$ isotope should be approximately $5 \%$ of the intensity of the monoisotopic peak. Mirrored with the unlabeled mass spectrum is the isotope distribution from the labeled experiment. Here, we see that that a peak corresponding to ${ }^{15} \mathrm{~N}$ enriched glutamate has taken the place of the ${ }^{13} \mathrm{C}$ glutamate signal. While the mass difference 
between the two isotopes is greater than $20 \mathrm{ppm}$, the low intensity of the ${ }^{13} \mathrm{C}$ isotope signal compromises mass accuracy and becomes masked by the ${ }^{15} \mathrm{~N}$ isotope signal.

In contrast to what we found in the $\mathrm{RCH} 2$ strain, it is apparent that the presence of ammonia does not suppress the activity of assimilatory nitrite reductase in the Oak Ridge strains to the same intense degree as in the case of $\mathrm{RCH} 2$. The data show that both ${ }^{15} \mathrm{~N}$ labeled and unlabeled metabolites are present in the strains isolated from Oak Ridge, indicating that these cells are utilizing both nitrogen sources for nitrogen assimilation. This finding was unexpected, as it would seem frivolous to generate ammonia when a sufficient supply was available. Indeed, it may be that competition for ammonia in the community that these cells were isolated from is particularly intense, meaning that these cells may have shifted toward nitrate assimilation to continually supplement the nitrogen source and compensate for general limited ammonia availability.

However, a recent study has shown that N2A2 and N2E2 differ from RCH2 in two very specific ways, which may explain the coutilization of ammonia and nitrate for assimilation. ${ }^{46}$ The strain $\mathrm{N} 2 \mathrm{~A} 2$ is similar to the $\mathrm{RCH} 2$ strain in that it contains the full denitrification pathway where nitrate is converted to nitrite and eventually becomes nitrogen gas. This study found that when N2A2 undergoes denitrification, nitrite tends to build up in the presence of groundwater contamination. The proposed reason for this accumulation is that the reduction of nitrate is more efficient than the reduction of nitrite, thus it is advantageous to exhaust the nitrate supply before beginning to reduce to nitrite.

Unfortunately, this preference for nitrate reduction leads to an accumulation of nitrite, which is toxic to the cells. $\mathrm{RCH} 2$ maintains low nitrite levels by reducing nitrite and nitrate simultaneously; this is not the case for N2A2. Since we have shown that N2A2 cells assimilate nitrate, we know that they have functioning assimilatory nitrite reductase, which may serve to lower the levels of toxic nitrite. In the case of N2E2, the ANRA pathway is also activated in the presence of ammonia but in this case has no choice since the denitrification pathway is incomplete, specifically missing dissimilatory nitrite reductase. Again, we posit that the nitrate assimilation pathway is activated, even in the presence of ammonia, to process the excess amount of nitrite produced during denitrification. These proposed mechanisms are shown in Figure 4.

\section{Multigroup Analysis of Nitrate Utilization}

To shed some light on the observed differences in nitrate utilization shown by global stable isotope metabolomics, we carried out a multigroup statistical analysis of the three bacterial metabolomes grown under the same conditions with both ammonia and nitrate present. The principal component analysis (Figure 5a) shows that the Oak Ridge strains form two groups close together that are distinctly separated from the Hanford strain. This indicates that the Hanford and Oak Ridge strains are metabolically different under control conditions. This was not unexpected, as we have determined that the N2A2 and N2E2 strains undergo coutilization of nitrate and ammonia while $\mathrm{RCH} 2$ does not.

A subset of the identified metabolites that contribute to this metabolic difference is shown in the box plots (Figure $5 \mathrm{~b}$ and $\mathrm{c}$ ). We found that purines and amino acids are anticorrelated between the Hanford and the Oak Ridge strains. In both cases, this is most likely due to the 
increased energy required to assimilate nitrate through the ANRA pathway. The accumulation of purines in the Oak Ridge strains reflects the increased energy requirement as well as the value of nitrogen to this system. Instead of degrading purines containing five nitrogens only to be recovered via de novo synthesis, these cells recycle purines through salvage pathways. ${ }^{11,47}$ Additionally, the low levels of amino acids are probably a direct result of the higher energy expense of assimilating nitrate. Indeed, it appears that the Oak Ridge strains have made downstream modifications in metabolism to accommodate the need to clear toxic nitrite though assimilation.

\section{Metabolite Isotope Pattern Analysis}

Using global isotope metabolomics, we also examined the nucleotide bases adenine and guanine to determine if a salvage pathway was evident in the isotope distribution measured in the Oak Ridge isolates. Mirror plots comparing the predicted isotope pattern for adenine and guanine to the measured isotope patterns were prepared to illustrate this difference (Figure 6). The predicted isotopic ratios were generated for each metabolite assuming a normal distribution of nitrogen atoms where $30 \%$ were ${ }^{15} \mathrm{~N}$. This is based on the percent of labeled glutamate measured in these experiments. Glutamate, which is being synthesized continuously, ${ }^{48}$ should represent the ratio at which ammonia and nitrate are being assimilated (70:30 ammonia/nitrate). The isotope distributions observed for the purines revealed that labeled nitrogen was approximately $15 \%$, which is about half the labeled nitrogen observed for glutamate, indicating an alternate source of unlabeled nitrogen must be responsible for the remaining purine biosynthesis. Thus, the isotope ratio shows that $d e$ novo synthesis would also be reduced by half ( $70 \%$ to $35 \%$ ) while the remaining $50 \%$ of the purines are expected to be generated via a salvage pathway (35:15:50 ammonia/nitrate/ salvage). In the case of nucleotide synthesis, it may be energetically favorable for these cells to recycle these molecules as they contain five atoms of nitrogen, as opposed to creating new molecules via de novo synthesis.

A discrepancy was also observed between amino acid and branched amino acids. The mirror plots (Figure 6) also show predicted and measured isotopic distributions for aspartate and valine. We found that aspartate tended to match the predicted pattern while valine was lower than expected. Again, this was taken as evidence of nitrogen economy dictated by the utilization of the nitrate assimilation by these cells. Branched amino acid biosynthesis is by its nature more complicated, and these cells may tend to degrade these valuable nitrogencontaining molecules at a slower rate.

\section{Nitrate Assimilation Pathway Analysis}

Global isotope metabolomics was developed using ${ }^{15} \mathrm{~N}$ labeled precursors to compare nitrogen metabolism across three Pseudomonas strains. Ammonia was removed from these cells to induce nitrate assimilation, which is typically blocked by the presence of ammonium. ${ }^{11}$ To understand how these nitrogen sources were being utilized, we employed global stable isotope metabolomics with ${ }^{15} \mathrm{~N}$ nitrate finding that N2A2 and N2E2 utilize the nitrate assimilation pathway in addition to assimilating ammonia. Since ammonia has been found to undergo half maximal labeling into E. coli metabolomes between 10 and $300 \mathrm{~s}$, ${ }^{49}$ the experimental labeling time of $\sim 10 \mathrm{~h}$ is likely more than adequate to reach a steady state 
of ${ }^{15} \mathrm{~N}$-labeled nitrate in the Pseudomonas metabolome. Furthermore, we observed that some nitrogen containing metabolites are labeled to different degrees, indicating differential rates of degradation and salvage. We hypothesize that this adaptation by the Oak Ridge strains is to increase conservation of nitrogen metabolites within their environment. This provides an overall snapshot of ${ }^{15} \mathrm{NO}_{3}$ incorporation into the Pseudomonas metabolome and therefore a gives an unbiased overview of the nitrate assimilation pathway. Further quantitative analysis of these metabolites and deeper insight into the ANRA pathway, metabolite salvage, and energy conservation would require a more rigorous experimental design including kinetic flux and mass-ratio based analysis to account for matrix interference and isotope incorporation distributions. ${ }^{41,42,49,50}$

All three strains tested here have homologues of the genes for the two-component regulatory nitrogen control system that activates expression of nitrate assimilation genes under conditions of nitrogen limitation. It is generally assumed that soil microorganisms use ammonia preferentially to nitrate as a nitrogen source. ${ }^{11}$ However, as we have observed in this report, this is not always the case. In a study of isolated soil microorganisms, ammonium was shown to severely inhibit nitrate assimilation for a Saccharomyces cerevisiae isolate and a Pseudomonas fluorescens isolate; however, an Azotobacter vinelandii isolate that was tested was not affected by ammonium. ${ }^{12}$ In Pseudomonas aeruginosa, $\mathrm{NtrBC}$ was shown to form a network with $\mathrm{CbrAB}$, another two component regulatory system involved in carbon source utilization to control $\mathrm{C} / \mathrm{N}$ balance. ${ }^{13}$ Deletion of NtrBC resulted in an inability to use nitrate, nitrite, and urea as sole nitrogen sources, while a constitutive allele of $n t r B$ impaired the growth of a strain lacking $\mathrm{CbrAB}$ on several carbon sources when ammonia was the nitrogen source. ${ }^{13}$ Under the conditions tested, N2A2 and N2E2 continued to use nitrate in the presence of ammonia; therefore these strains may still use NtrBC to control nitrogen balance in conjunction with limited availability of complex carbon sources.

The pristine well that the Oak Ridge strains were isolated from (FW300) was measured to have $0.005 \pm 0.001 \mathrm{mM}$ nitrate and $0.033 \pm 0.006 \mathrm{mM}$ ammonia. A potential source of nitrate in the noncontaminated region of Oak Ridge is leaf litter. Pseudomonas syringae pv Syringae B728a has been shown to use nitrate as a nitrogen source when cultured on leaves as a mutant strain lacking NasB was greatly inhibited in growth. ${ }^{51}$ This coutilization may have been adapted for several reasons, for example these bacteria live in complex communities where there is possible competition for the readily assimilated nitrogen source and a readily reduced electron acceptor (nitrate). Likewise, the strains from Oak Ridge differ from the $\mathrm{RCH} 2$ strain in that the denitrification pathways are predisposed for the accumulation of nitrite, which is toxic. As a result of the nitrate assimilation pathway producing nitrite as an intermediate, assimilation may use this pathway to lower toxic nitrite levels.

The use of global isotope metabolomics has allowed us to discover unexpected coutilization of nitrate and ammonia, which would have been impossible to observe through standard global metabolomic methods. Global isotope metabolomics has added an informative second dimension by identifying specific pathways utilized by each strain. The approach will be used in the future to probe the fundamentals of nitrogen metabolism under specific 
environmental conditions, including the role of agricultural products on the symbiotic relationship between plants and their microbiota.

\section{METHODS}

\section{Pseudomonas Growth Conditions}

The basal growth medium had the following composition: $20 \mathrm{mM}$ sodium fumarate, $1.3 \mathrm{mM}$ $\mathrm{KCl}, 2 \mathrm{mM} \mathrm{MgSO}_{4}, 0.2 \mathrm{mM} \mathrm{NaCl}, 1.2 \mathrm{mM} \mathrm{NaHCO}_{3}$, and $5 \mathrm{mM} \mathrm{NaH}_{2} \mathrm{PO}_{4}$ with sterile vitamin and trace elements prepared as described by Widdel and Bak. ${ }^{52}$ As nitrogen sources, $\mathrm{KNO}_{3}, \mathrm{~K}^{15} \mathrm{NO}_{3}$ (Sigma-Aldrich), and $\mathrm{NH}_{4} \mathrm{Cl}$ were used at the indicated concentrations. Initial cultures were grown aerobically in the basal medium with $5 \mathrm{mM} \mathrm{NH}_{4} \mathrm{Cl}$. These were then centrifuged and washed three times in basal medium lacking a nitrogen source and were then diluted 20 -fold into the experimental growth medium containing $5 \mathrm{mM}$ ammonia with or without $10 \mathrm{mM}$ unlabeled nitrate or $10 \mathrm{mM}{ }^{15} \mathrm{~N}$ labeled nitrate. Experimental cultures $(6 \mathrm{~mL})$ were grown anaerobically under a $100 \%$ argon atmosphere in $15 \mathrm{~mL}$ Balch tubes at $30{ }^{\circ} \mathrm{C}$ with continuous shaking. Cells were grown until the late log phase as determined by OD (typically 10-11 h) and were harvested by centrifugation for $2 \mathrm{~min}$ at 14 $000 \mathrm{rpm}$ before flash freezing in liquid nitrogen. All experiments were performed in biological quintuplicate. Protein concentrations were determined by the Bradford method ${ }^{53}$ and used to normalize the volume for extract reconstitution as discussed below.

\section{Metabolite Extraction}

The metabolites were extracted from cell pellets by a methanol:acetonitrile:water $(2: 2: 1, \mathrm{v} / \mathrm{v})$ solvent mixture. An adjusted volume of $1 \mathrm{~mL}$ of cold solvent was added to each pellet, vortexed for $30 \mathrm{~s}$, and incubated in liquid nitrogen for $1 \mathrm{~min}$. The samples were then allowed to thaw at RT and then sonicated for $15 \mathrm{~min}$. To precipitate proteins, the samples were incubated for $1 \mathrm{~h}$ at $-20{ }^{\circ} \mathrm{C}$, followed by centrifugation at $16000 \mathrm{~g}$ and $4{ }^{\circ} \mathrm{C}$ for $15 \mathrm{~min}$. The resulting supernatant was removed and evaporated to dryness in a vacuum concentrator (LABCONCO CentriVap Benchtop). The dry extracts were then reconstituted in the appropriate volume of acetonitrile/water $(1: 1, \mathrm{v} / \mathrm{v})$, scaled to protein concentration with the lowest concentration $=100 \mu \mathrm{L}$, sonicated for $10 \mathrm{~min}$, and centrifuged for $15 \mathrm{~min}$ at $16000 \mathrm{~g}$ and $4{ }^{\circ} \mathrm{C}$ to remove insoluble debris. The supernatants were transferred to HPLC vials and stored at $-80^{\circ} \mathrm{C}$ prior to $\mathrm{LC} / \mathrm{MS}$ analysis.

\section{LC/MS Analysis}

Cell extracts were analyzed on a 6550 iFunnel QTOF mass spectrometer (Agilent Technologies) interfaced with a 1290 UPLC system (Agilent Technologies). HPLC was carried out on a Luna Aminopropyl, $3 \mu \mathrm{m}, 150 \mathrm{~mm} \times 1.0 \mathrm{~mm}$ I.D. HILIC column (Phenomenex). The mobile phase was composed of $\mathrm{A}=20 \mathrm{mM}$ ammonium acetate and 40 $\mathrm{mM}$ ammonium hydroxide in $95 \%$ water (all \% were v/v unless otherwise indicated) and B $=95 \%$ acetonitrile. The remaining $5 \%$ components were acetonitrile or water, respectively.

A linear gradient from $100 \%$ B (0-5 min) to $100 \%$ A (50-55 min) was applied. A 10 min reequilibration time was applied to the HILIC column for re-equilibration and maintenance of reproducibility. The flow rate was $50 \mu \mathrm{L} / \mathrm{min}$, and the sample injection volume was $5 \mu \mathrm{L}$. ESI source conditions were set as follows: dry gas temperature, $200{ }^{\circ} \mathrm{C}$; flow, $11 \mathrm{~L} / \mathrm{min}$, 
fragmentor, $380 \mathrm{~V}$; sheath gas temperature, $300{ }^{\circ} \mathrm{C}$; flow, $9 \mathrm{~L} / \mathrm{min}$; nozzle voltage, $500 \mathrm{~V}$; capillary voltage, $-2500 \mathrm{~V}$ in ESI negative mode. The instrument was set to acquire data over the $\mathrm{m} / \mathrm{z}$ range $50-1000$, with the MS acquisition rate of 1.6 spectra/s. The sample sequence was randomized to avoid systematic decreases in signals over sample sets.

\section{Data Processing}

Statistical analysis of global metabolomic data was performed by XCMS Online using twosample Mann-Whitney $t$ tests and using the following parameters: ppm $=20$; RT window $=$ $10 \mathrm{~s}$; fold change $>1.5 ; p$ value $<0.01$; max intensity $>10000$. For targeted analysis, the Student's $t$ test was performed for unpaired data on each Pseudomonas strain, in the presence and absence of ammonium. Isotopically labeled samples were analyzed using the $\mathrm{X}^{13} \mathrm{CMS}$ software package ${ }^{43}$ to identify isotope labeling patterns. The following parameters were employed: mass of ${ }^{14} \mathrm{~N}=14.003074$; mass difference between ${ }^{15} \mathrm{~N}$ and ${ }^{14} \mathrm{~N}=$ 0.997035; noiseCutoff $=10000$ ion counts. Cloud plots and heat maps were generated via $\mathrm{R}$ scripts developed in-house and integrated into XCMS. Statistical PCA was performed using the R pcaMethods package, also integrated into XCMS.

\section{Strain Isolation and Sequencing}

Strain N2A2 was previously isolated from groundwater collected from well FW300 on the Oak Ridge Reservation and was cultured on a basal medium (with $10 \mathrm{mM}$ sodium acetate as the sole carbon source and electron donor and $10 \mathrm{mM}$ sodium nitrate as the electron acceptor). Strain N2E2 was isolated from groundwater collected from the same well on the same basal medium, but with $10 \mathrm{mM}$ sodium propionate as the sole carbon source and electron donor and $10 \mathrm{mM}$ sodium nitrate as the electron acceptor. ${ }^{46}$ The completed or draft genome sequences for each strain are available in the Integrated Microbial Genomes system (http://genome.jgi.doe.gov/): RCH2 (2507149015), N2A2 (2600255294), and N2E2 (2600255295).

\section{Supplementary Material}

Refer to Web version on PubMed Central for supplementary material.

\section{Acknowledgments}

The authors thank the following for funding assistance: Ecosystems and Networks Integrated with Genes and Molecular Assemblies (http://enigma.lbl.gov), a Scientific Focus Area Program at Lawrence Berkeley National Laboratory for the U.S. Department of Energy, Office of Science, Office of Biological and Environmental Research under contract number DE-AC02-05CH11231, and the National Institutes of Health (NIH) grants R01 GMH4368 and PO1 A1043376-02S1.

\section{References}

1. Canfield DE, Glazer AN, Falkowski PG. The Evolution and Future of Earth's Nitrogen Cycle. Science. 2010; 330:192-196. [PubMed: 20929768]

2. Vitousek PM, Menge DNL, Reed SC, Cleveland CC. Biological nitrogen fixation: rates, patterns and ecological controls in terrestrial ecosystems. Philos Trans R Soc, B. 2013; 368:9.

3. Green PA, Vorosmarty CJ, Meybeck M, Galloway JN, Peterson BJ, Boyer EW. Pre-industrial and contemporary fluxes of nitrogen through rivers: a global assessment based on typology.

Biogeochemistry. 2004; 68:71-105. 
4. Diaz RJ, Rosenberg R. Spreading dead zones and consequences for marine ecosystems. Science. 2008; 321:926-929. [PubMed: 18703733]

5. Howarth R, Chan F, Conley DJ, Garnier J, Doney SC, Marino R, Billen G. Coupled biogeochemical cycles: eutrophication and hypoxia in temperate estuaries and coastal marine ecosystems. Frontiers in Ecology and the Environment. 2011; 9:18-26.

6. Vaquer-Sunyer R, Conley DJ, Muthusamy S, Lindh MV, Pinhassi J, Kritzberg ES. Dissolved Organic Nitrogen Inputs from Wastewater Treatment Plant Effluents Increase Responses of Planktonic Metabolic Rates to Warming. Environ Sci Technol. 2015; 49:11411-11420. [PubMed: 26356812]

7. Paerl HW. Cultural eutrophication of shallow coastal waters: Coupling changing anthropogenic nutrient inputs to regional management approaches. Limnologica. 1999; 29:249-254.

8. Vaccaro BJ, Thorgersen MP, Lancaster WA, Price MN, Wetmore KM, Poole FL, Deutschbauer A, Arkin AP, Adams MWW. Determining Roles of Accessory Genes in Denitrification by Mutant Fitness Analyses. Appl Environ Microbiol. 2016; 82:51-61.

9. Richardson D, Berks B, Russell D, Spiro S, Taylor C. Functional, biochemical and genetic diversity of prokaryotic nitrate reductases. Cell Mol Life Sci. 2001; 58:165-178. [PubMed: 11289299]

10. Stolz JF, Basu P. Evolution of nitrate reductase: Molecular and structural variations on a common function. Chem Bio Chem. 2002; 3:198-206.

11. Merrick MJ, Edwards RA. Nitrogen Control in Bacteria. Microbiol Rev. 1995; 59:604-622. [PubMed: 8531888]

12. Rice CW, Tiedje JM. Regulation of nitrate assimilation by ammonium in soils and in isolated soil microorganisms. Soil Biol Biochem. 1989; 21:597-602.

13. Li W, Lu CD. Regulation of carbon and nitrogen utilization by CbrAB and NtrBC two-component systems in Pseudomonas aeruginosa. Journal of bacteriology. 2007; 189:5413-5420. [PubMed: 17545289]

14. Luque-Almagro VM, Gates AJ, Moreno-Vivian C, Ferguson SJ, Richardson DJ, Roldan MD. Bacterial nitrate assimilation: gene distribution and regulation. Biochem Soc Trans. 2011; 39:1838-1843. [PubMed: 22103536]

15. Nicholson JK, Lindon JC, Holmes E. 'Metabonomics': understanding the metabolic responses of living systems to pathophysiological stimuli via multivariate statistical analysis of biological NMR spectroscopic data. Xenobiotica. 1999; 29:1181-1189. [PubMed: 10598751]

16. Serkova NJ, Niemann CU. Pattern recognition and biomarker validation using quantitative H-1NMR-based metabolomics. Expert Rev Mol Diagn. 2006; 6:717-731. [PubMed: 17009906]

17. Bertini I, Hu XY, Luchinat C. Global metabolomics characterization of bacteria: pre-analytical treatments and profiling. Metabolomics. 2014; 10:241-249.

18. Fiehn O, Kopka J, Dormann P, Altmann T, Trethewey RN, Willmitzer L. Metabolite profiling for plant functional genomics. Nat Biotechnol. 2000; 18:1157-1161. [PubMed: 11062433]

19. Villas-Boas SG, Hojer-Pedersen J, Akesson M, Smedsgaard J, Nielsen J. Global metabolite analysis of yeast: evaluation of sample preparation methods. Yeast. 2005; 22:1155-1169. [PubMed: 16240456]

20. Pasikanti KK, Esuvaranathan K, Ho PC, Mahendran R, Kamaraj R, Wu QH, Chiong E, Chan ECY. Noninvasive Urinary Metabonomic Diagnosis of Human Bladder Cancer. J Proteome Res. 2010; 9:2988-2995. [PubMed: 20337499]

21. Wikoff WR, Anfora AT, Liu J, Schultz PG, Lesley SA, Peters EC, Siuzdak G. Metabolomics analysis reveals large effects of gut microflora on mammalian blood metabolites. Proc Natl Acad Sci U S A. 2009; 106:3698-3703. [PubMed: 19234110]

22. Creek DJ, Jankevics A, Breitling R, Watson DG, Barrett MP, Burgess KEV. Toward Global Metabolomics Analysis with Hydrophilic Interaction Liquid Chromatography-Mass Spectrometry: Improved Metabolite Identification by Retention Time Prediction. Anal Chem. 2011; 83:87038710. [PubMed: 21928819]

23. Ankrah NYD, May AL, Middleton JL, Jones DR, Hadden MK, Gooding JR, LeCleir GR, Wilhelm SW, Campagna SR, Buchan A. Phage infection of an environmentally relevant marine bacterium alters host metabolism and lysate composition. ISME J. 2014; 8:1089-1100. [PubMed: 24304672] 
24. Fei F, Bowdish DME, McCarry BE. Comprehensive and simultaneous coverage of lipid and polar metabolites for endogenous cellular metabolomics using HILIC-TOF-MS. Anal Bioanal Chem. 2014; 406:3723-3733. [PubMed: 24714971]

25. Yin PY, Xu GW. Current state-of-the-art of nontargeted metabolomics based on liquid chromatography-mass spectrometry with special emphasis in clinical applications. J Chromatogr A. 2014; 1374:1-13. [PubMed: 25444251]

26. Lommen A. MetAlign: interface-driven, versatile metabolomics tool for hyphenated full-scan mass spectrometry data preprocessing. Anal Chem. 2009; 81:3079-3086. [PubMed: 19301908]

27. Pluskal T, Castillo S, Villar-Briones A, Oresic M. MZmine 2: modular framework for processing, visualizing, and analyzing mass spectrometry-based molecular profile data. BMC Bioinf. 2010; 11:395.

28. Melamud E, Vastag L, Rabinowitz JD. Metabolomic analysis and visualization engine for LC-MS data. Anal Chem. 2010; 82:9818-9826. [PubMed: 21049934]

29. Xia J, Mandal R, Sinelnikov IV, Broadhurst D, Wishart DS. MetaboAnalyst 2.0-a comprehensive server for metabolomic data analysis. Nucleic Acids Res. 2012; 40:W127-133. [PubMed: 22553367]

30. Smith CA, Want EJ, O’Maille G, Abagyan R, Siuzdak G. XCMS: Processing mass spectrometry data for metabolite profiling using Nonlinear peak alignment, matching, and identification. Anal Chem. 2006; 78:779-787. [PubMed: 16448051]

31. Tautenhahn R, Patti GJ, Rinehart D, Siuzdak G. XCMS Online: A Web-Based Platform to Process Untargeted Metabolomic Data. Anal Chem. 2012; 84:5035-5039. [PubMed: 22533540]

32. Gowda H, Ivanisevic J, Johnson CH, Kurczy ME, Benton HP, Rinehart D, Nguyen T, Ray J, Kuehl J, Arevalo B, Westenskow PD, Wang JH, Arkin AP, Deutschbauer AM, Patti GJ, Siuzdak G. Interactive XCMS Online: Simplifying Advanced Metabolomic Data Processing and Subsequent Statistical Analyses. Anal Chem. 2014; 86:6931-6939. [PubMed: 24934772]

33. Smith CA, O'Maille G, Want EJ, Qin C, Trauger SA, Brandon TR, Custodio DE, Abagyan R, Siuzdak G. METLIN - A metabolite mass spectral database. Ther Drug Monit. 2005; 27:747-751. [PubMed: 16404815]

34. Kurczy ME, Ivanisevic J, Johnson CH, Uritboonthai W, Hoang L, Fang M, Hicks M, Aldebot A, Rinehart D, Mellander LJ. Determining conserved metabolic biomarkers from a million database queries. Bioinformatics. 2015:btv475.

35. Kanehisa M, Goto S, Kawashima S, Okuno Y, Hattori M. The KEGG resource for deciphering the genome. Nucleic Acids Res. 2004; 32:D277-D280. [PubMed: 14681412]

36. Wishart DS, Jewison T, Guo AC, Wilson M, Knox C, Liu YF, Djoumbou Y, Mandal R, Aziat F, Dong E, Bouatra S, Sinelnikov I, Arndt D, Xia JG, Liu P, Yallou F, Bjorndahl T, Perez-Pineiro R, Eisner R, Allen F, Neveu V, Greiner R, Scalbert A. HMDB 3.0-The Human Metabolome Database in 2013. Nucleic Acids Res. 2013; 41:D801-D807. [PubMed: 23161693]

37. Cui Q, Lewis IA, Hegeman AD, Anderson ME, Li J, Schulte CF, Westler WM, Eghbalnia HR, Sussman MR, Markley JL. Metabolite identification via the Madison Metabolomics Consortium Database. Nat Biotechnol. 2008; 26:162-164. [PubMed: 18259166]

38. Goldstein JL, Ho YK, Basu SK, Brown MS. Binding-Site on Macrophages that Mediates Uptake and Degradation of Acetylated Low-Density Lipoprotein, Producing Massive Cholesterol Deposition. Proc Natl Acad Sci U S A. 1979; 76:333-337. [PubMed: 218198]

39. Hellerstein MK, Neese RA. Mass Isotopomer Distribution Analysis - A Technique for Measuring Biosynthesis and Turnover of Polymers. Am J Physiol. 1992; 263:E988-E1001. [PubMed: 1443132]

40. Locasale JW, Grassian AR, Melman T, Lyssiotis CA, Mattaini KR, Bass AJ, Heffron G, Metallo CM, Muranen T, Sharfi H, Sasaki AT, Anastasiou D, Mullarky E, Vokes NI, Sasaki M, Beroukhim R, Stephanopoulos G, Ligon AH, Meyerson M, Richardson AL, Chin L, Wagner G, Asara JM, Brugge JS, Cantley LC, Vander Heiden MG. Phosphoglycerate dehydrogenase diverts glycolytic flux and contributes to oncogenesis. Nat Genet. 2011; 43:869-U879. [PubMed: 21804546]

41. Yuan J, Bennett BD, Rabinowitz JD. Kinetic flux profiling for quantitation of cellular metabolic fluxes. Nat Protoc. 2008; 3:1328-1340. [PubMed: 18714301] 
42. Bennett BD, Yuan J, Kimball EH, Rabinowitz JD. Absolute quantitation of intracellular metabolite concentrations by an isotope ratio-based approach. Nat Protoc. 2008; 3:1299-1311. [PubMed: $18714298]$

43. Huang XJ, Chen YJ, Cho K, Nikolskiy I, Crawford PA, Patti GJ. XCMS)-C-13: Global Tracking of Isotopic Labels in Untargeted Metabolomics. Anal Chem. 2014; 86:1632-1639. [PubMed: 24397582]

44. Cho K, Mahieu N, Ivanisevic J, Uritboonthai W, Chen YJ, Siuzdak G, Patti GJ. isoMETLIN: A Database for Isotope-Based Metabolomics. Anal Chem. 2014; 86:9358-9361. [PubMed: 25166490]

45. Gika HG, Theodoridis GA, Vrhovsek U, Mattivi F. Quantitative profiling of polar primary metabolites using hydrophilic interaction ultrahigh performance liquid chromatography-tandem mass spectrometry. J Chromatogr A. 2012; 1259:121-127. [PubMed: 22381888]

46. Thorgersen MP, Lancaster WA, Vaccaro BJ, Poole FL, Rocha AM, Mehlhorn T, Pettenato A, Ray J, Waters RJ, Melnyk RA, Chakraborty R, Hazen TC, Deutschbauer AM, Arkin AP, Adams MWW. Molybdenum Availability Is Key to Nitrate Removal in Contaminated Groundwater Environments. Appl Environ Microbiol. 2015; 81:4976-4983. [PubMed: 25979890]

47. Beck DA, O’Donovan GA. Pathways of pyrimidine salvage in Pseudomonas and former Pseudomonas: Detection of recycling enzymes using high-performance liquid chromatography. Curr Microbiol. 2008; 56:162-167. [PubMed: 17962997]

48. Frimmersdorf E, Horatzek S, Pelnikevich A, Wiehlmann L, Schomburg D. How Pseudomonas aeruginosa adapts to various environments: a metabolomic approach. Environ Microbiol. 2010; 12:1734-1747. [PubMed: 20553553]

49. Yuan J, Fowler WU, Kimball E, Lu WY, Rabinowitz JD. Kinetic flux profiling of nitrogen assimilation in Escherichia coli. Nat Chem Biol. 2006; 2:529-530. [PubMed: 16936719]

50. Yuan J, Rabinowitz JD. Differentiating metabolites formed from de novo synthesis versus macromolecule decomposition. J Am Chem Soc. 2007; 129:9294-9295. [PubMed: 17616198]

51. Parangan-Smith A, Lindow S. Contribution of nitrate assimilation to the fitness of Pseudomonas syringae pv. syringae B728a on plants. Appl Environ Microbiol. 2013; 79:678-687. [PubMed: 23160124]

52. Widdel, F., Bak, F. The prokaryotes. Springer; 1992. Gram-negative mesophilic sulfate-reducing bacteria; p. 3352-3378.

53. Bradford MM, Williams WL. New, Rapid, Sensitive Method for Protein Determination. Fed Proc. 1976; 35:274-274. 

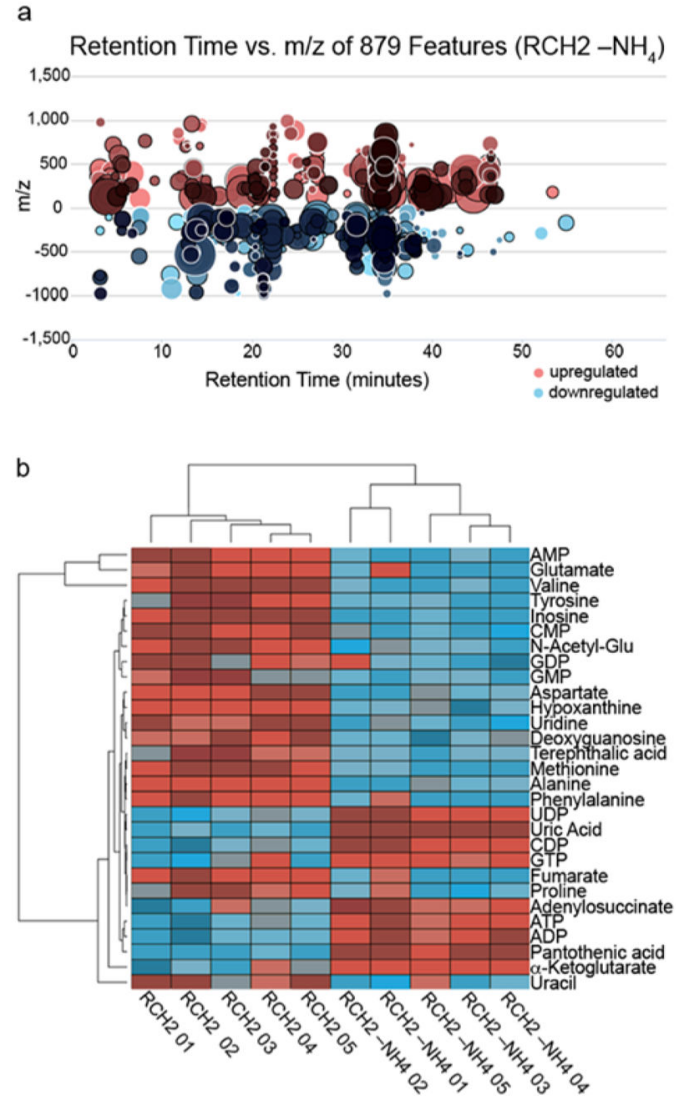

Figure 1.

RCH2 metabolome under ammonia-free conditions. (a) A cloud plot showing 879 altered metabolite features where red is increased and blue is decreased. Each circle represents a unique $\mathrm{m} / \mathrm{z}$ retention time signal where a darker color represents a lower $p$ value and the diameter scales with the fold change. The cutoffs for this cloud plot were as follows ( $p$ value $<0.01$, fold change $>1.5$, intensity $>10000$ ). (b) A heat map and dendogram showing dysegulated metabolites that were identified by tandem MS. Five replicates 01 through 05 for both $\mathrm{RCH} 2$ growth conditions, in the presence and absence $(-\mathrm{NH} 4)$ of ammonium, is labeled accordingly on the $x$ axis. The color intensity is proportional to the integrated signal intensity. 


\section{a}
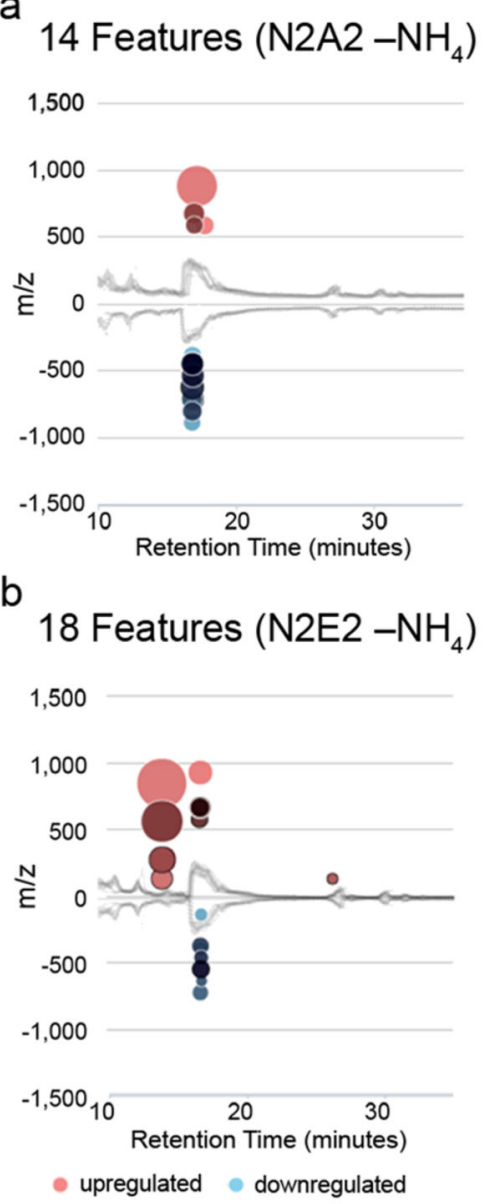

Figure 2.

N2A2 and N2E2 metabolome under ammonia-free conditions. (a) A cloud plot showing 14 altered metabolite features for N2A2 under ammonia-free conditions and (b) a cloud plot showing 18 altered metabolite features for N2E2 under ammonia-free conditions where red is upregulated and blue is downregulated. Each circle represents a unique $\mathrm{m} / z$ retention time signal where darker color represents a lower $p$ value and the diameter scales with the fold change. The cutoffs for this cloud plot were as follows: $p$ value $<0.01$, fold change $>1.5$, intensity $>10000$. 


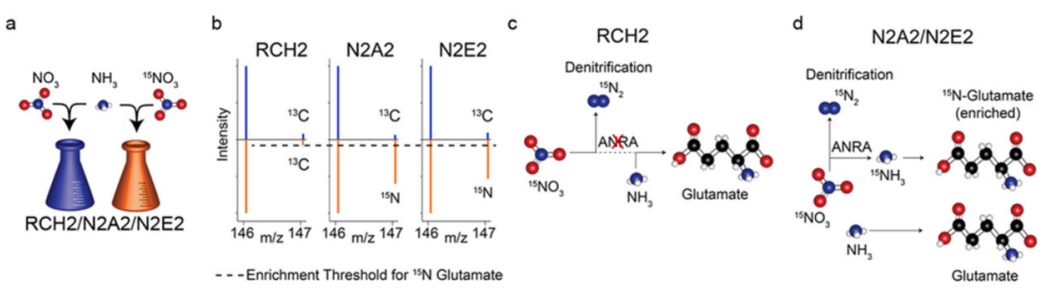

Figure 3.

Global ${ }^{15} \mathrm{~N}$-isotope metabolomics showing two separate nitrogen assimilation pathways. (a) Experimental conditions where each strain $(\mathrm{RCH} 2 / \mathrm{N} 2 \mathrm{~A} 2 / \mathrm{N} 2 \mathrm{E} 2)$ is grown in the presence of $5 \mathrm{mM}$ ammonium and $10 \mathrm{mM}$ nitrate or $10 \mathrm{mM}{ }^{15} \mathrm{~N}$-nitrate until the late log phase $(\sim 10 \mathrm{~h})$. (b) Comparison of isotope ratios measured for glutamate for each strain under the labeled and unlabeled conditions where blue is control and orange is labeled. (c) The nitrogen assimilation pathway used by the RCH2 strain. RCH2 exclusively uses ammonium for assimilation and does not utilize the Assimilatory Nitrate Reduction to Ammonia pathway (ANRA). Thus, only unlabeled glutamate is produced. This leaves nitrate available for denitrification. (d) Pathways utilized by N2A2/N2E2 for nitrogen assimilation. Both direct assimilation of ammonium and ANRA are utilized as both glutamate and enriched glutamate are detected. 

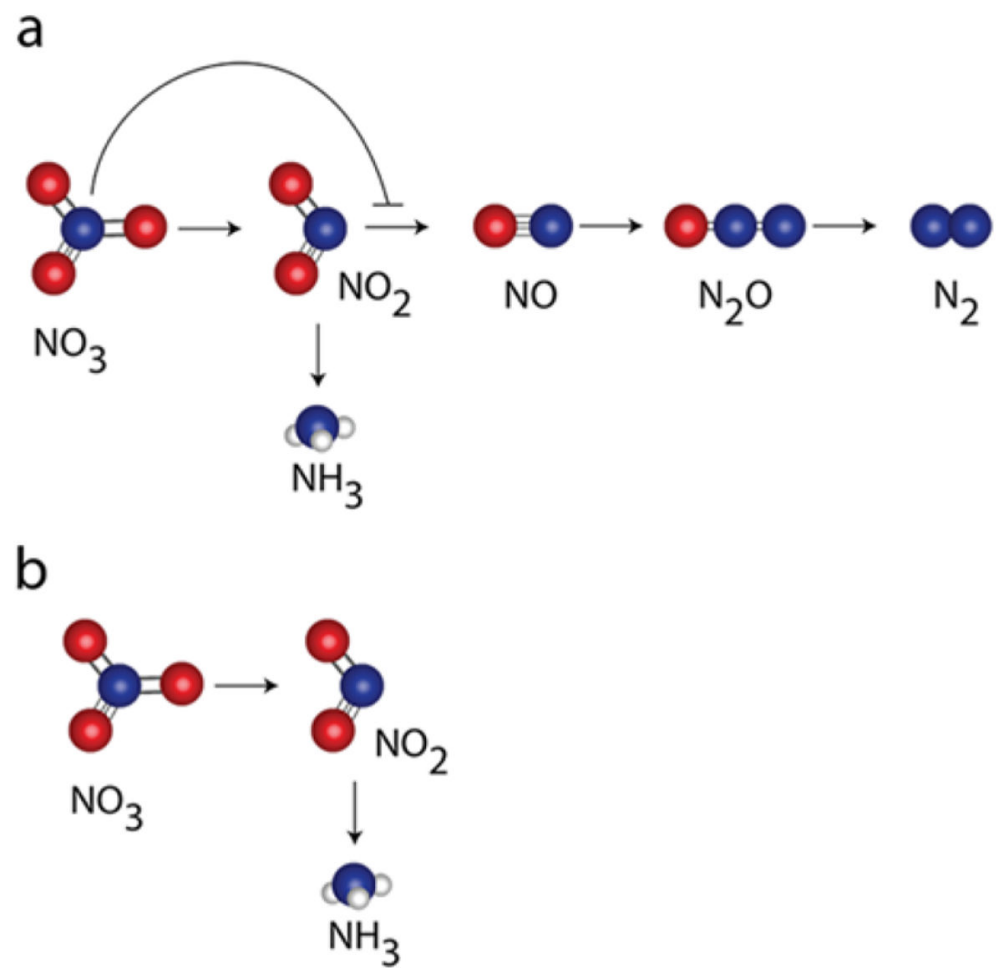

Figure 4.

Denitrification and nitrate assimilation pathway overlap. (a) Complete denitrification in $\mathrm{N} 2 \mathrm{~A} 2$ is impeded by $\mathrm{NO}_{3}$, which inhibits dissimilatory nitrite reductase; the accumulated nitrite is then reduced to ammonia by assimilatory nitrite reductase. (b) N2E2 lacks dissimilatory nitrite reductase, and the nitrite is reduced to ammonia by assimilatory nitrite reductase. 
a

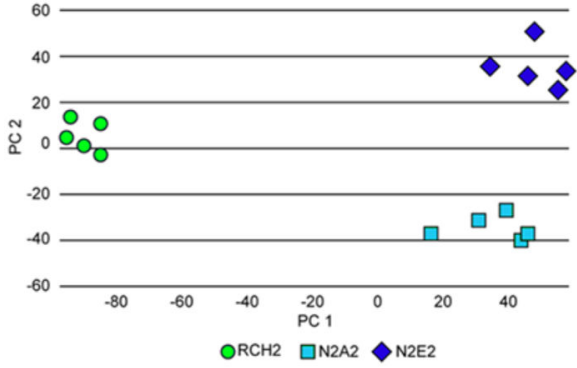

Hanford Oak Ridge Hanford Oak Ridge

b

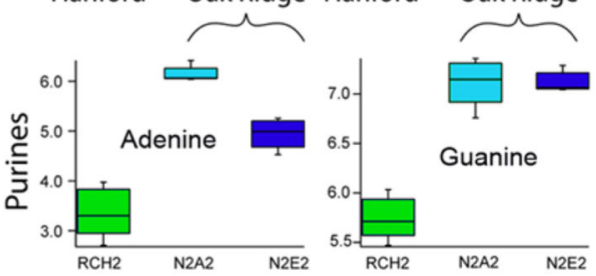

C

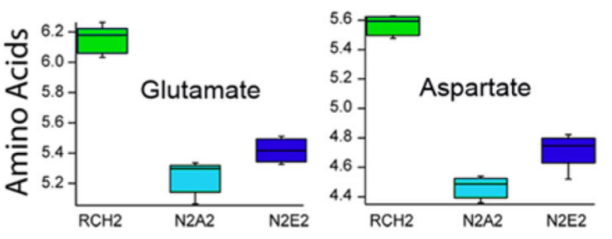

Figure 5.

Pseudomonas multigroup metabolomics analysis. (a) Principal component analysis of the metabolome of $\mathrm{RCH} 2, \mathrm{~N} 2 \mathrm{~A} 2$, and N2E2 grown in the presence of both nitrate and ammonia showing statistically significant separation between strains, particularly RCHS vs N2As and $\mathrm{N} 2 \mathrm{E} 2$. The percent variance for $\mathrm{PC} 1=47 \%$ and $\mathrm{PC} 2=8 \%$. (b) Comparison of purine levels and (c) selected amino acid levels across RCH2, N2A2, and N2E2 using box and whisker plots to illustrate the median metabolite concentration ( $\log 10$ [signal intensity]), upper and lower quartiles (box), and extremes (whiskers) of each sample set. Purines were upregulated in N2A2 and N2E2, which reflects the increased energy requirements, while amino acids were down regulated as an energy expenditure of assimilating nitrate. 

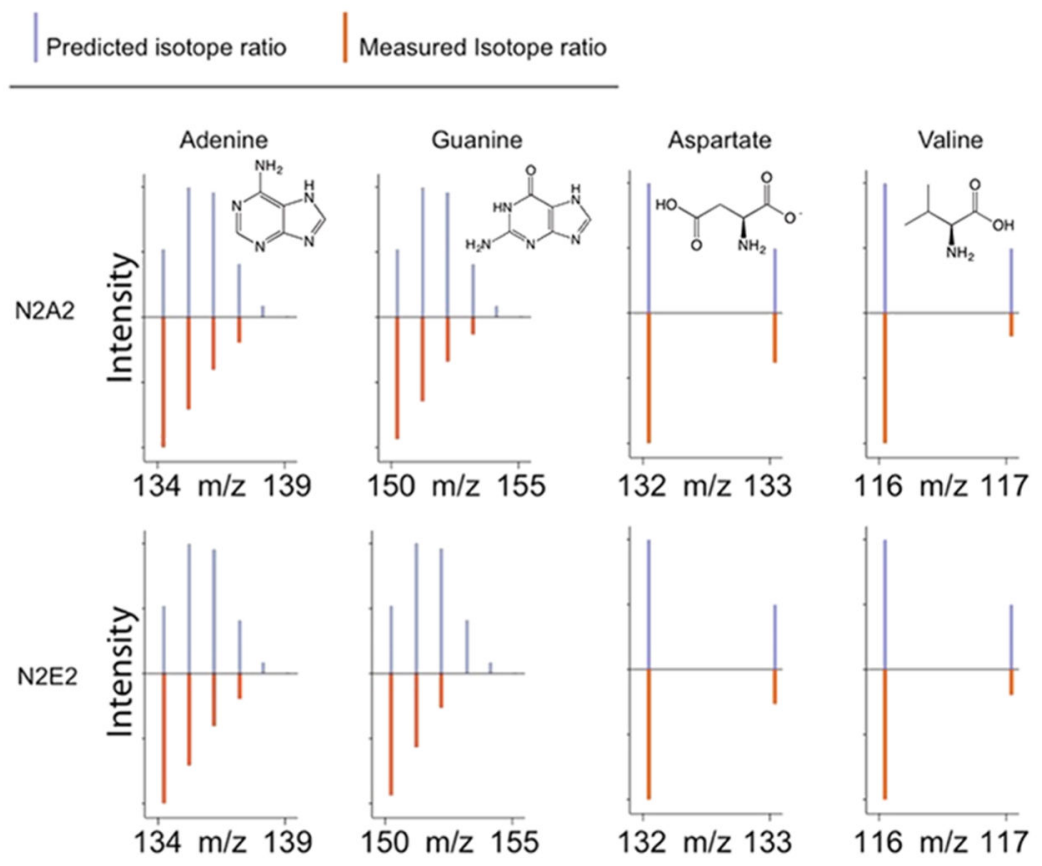

Figure 6.

Isotope pattern mirror plots. Predicted (blue, top) and measured (orange, bottom) isotope patterns for adenine, guanine, aspartate, and valine. The metabolites were found to have decreased quantities of incorporated ${ }^{15} \mathrm{~N}$ compared to the predicted isotope ratios based on a normal distribution of nitrogen atoms where $30 \%$ were ${ }^{15} \mathrm{~N}$. 